\title{
Root Cause Analysis of Congestion in Wireless Sensor Network
}

\author{
Vivek Deshpande \\ Dept. of Information Technology \\ MIT college of Engineering \\ Paud Road, Kothrud, Pune-38
}

\author{
Prachi Sarode \\ Dept. of Information Technology \\ MIT college of Engineering \\ Paud Road, Kothrud, Pune-38
}

\author{
Sambhaji Sarode \\ Dept. of Computer Engineering \\ MIT college of Engineering \\ Paud Road, Kothrud, Pune-38
}

\begin{abstract}
In wireless sensor networks data, which get generated, is not always same; some data may be more important than others and having different priorities. As deployment sizes and data rates grow, congestion becomes a major problem in these networks. The congestion results in arbitrary dropping of data packets that reduce the overall network throughput. In this paper, we discuss the various parameters (root causes of congestion), which help us to avoid and control the congestion in the wireless sensor network. The parameters consider in this paper are input/output flow rate, node density, non-linear or unbalanced distribution of load, processing / service time of node and reliability of network.
\end{abstract}

\section{Categories and Subject Descriptors}

Wireless Communication

\section{General Terms}

Algorithms, Reliability

\section{Keywords}

Wireless Sensor Network, Congestion control and avoidances.

\section{INTRODUCTION}

Wireless sensor Network consist of small microcontroller fitted with sensors and some means of communication radios. They are distributed over wide area and transmit gathered data to one or many central nodes called as Sink or also know as base station.

Wireless sensor network (WSN) is one of the emerging research areas that provide designated services such as disaster prevention, environment monitoring, medical monitoring, habitat monitoring, military surveillance, inventory tracking, intelligent logistics, and health monitoring. These networks deliver numerous types of traffic, from simple periodic reports to unpredictable bursts of messages due to this congestion control in wireless sensor network is very rare event. Congestion occurs in WSN due to i) Radio channel interference, ii) Addition and removal of sensor nodes, iii) Lastly sensed event cause bursts of messages.

WSNs have some problems to be overcome, for example energyconservation congestion control, reliability data dissemination, and security. These problems often involve in one or several layers top-down from application layer to physical layer, and can be studies separately in each corresponding layer, or collaboratively cross each layer. One of the important problem, congestion control may involve in only transport layer, but energy-conservation may be related to physical layer, data link layer, network layer, and high layers. Many researchers recently turn their attentions to transport layer protocols, which are important for reliable data dissemination and energy-conservation for WSNs.

Congestion causes many problems when sensors receives more packets than that its buffer space, the excess packets has to be dropped energy consumed by sensor nodes on the packet is wasted. And if further packet has traveled, the more waste is, which in turn diminish the network throughput and reliable data transmissions. Congestion control studies how to recover from congestion. Congestion avoidance studies how to prevent congestion from happening for this we have to monitor the parameters which can helps us to avoid congestion in WSN which is the subject of this paper.

The remainder of the paper is organized as follows. Section 2 lists existing congestion control protocol. Section 3 present parameters, which help to avoid congestion. And lastly Section 4 concludes the whole paper on the note of future work.

\section{RELATED WORK}

There are many transport layer protocol that describes the causes or reasons of congestion with reliable data transfer in upstream (from sensor node to sink) and downstream (from sink to sensor node) in wireless sensor network.

ESRT is an End-to-End approach, [3] which gives the guarantee of reliability by regulating sensor report frequency. It provides reliability for applications not for each single packet. It can control sensor report frequency. ESRT monitors the local buffer level of sensor nodes and sets a congestion notification bit in the packets it forwards to sinks if the buffer overflows. If a sink receives a packet with the congestion notification bit set it infers congestion and broadcasts a control signal informing all source nodes to reduce their common reporting frequency according to some function. ESRT brings with such disadvantages: 1) ESRT regulates report frequency of all sensors using the same value. But it may be more reasonable if using different value since each sensor may have different contributions to congestion. 2) ESRT assumes and uses a channel (one-hop) with high power that will influence the on-going data transmission. 3) ESRT mainly considers reliability and energy-conservation. 
CODA (Congestion Detection and Avoidance) [4] is another energy efficient congestion control scheme. It performs three mechanisms:

1. Congestion detection,

2. Open-loop hop-by-hop backpressure mechanism and

3. A close-loop multi-source regulation mechanism. CODA attempts to detect congestion by monitoring current buffer occupancy and wireless channel load. In an open-loop hop-by-hop backpressure if buffer occupancy or wireless channel load exceeds a threshold, it means that congestion has occurred. The node that has detected congestion will then notify its upstream neighbor to reduce its rate. Using AIMD method the upstream neighbor nodes trigger reduction of their output rate. CODA regulates a multisource rate through a closed-loop end-to-end approach, as follows: (1) When a sensor node exceeds its theoretical rate, it sets a "regulation" bit in the "event" packet; (2) If the event packet received by the sink has a "regulation" bit set, the sink sends an ACK message to the sensor nodes and informs them to reduce their rate; and (3) if the congestion is cleared, the sink will send an immediate ACK control message to the sensor nodes, informing them that they can increase their rate. CODA has some disadvantages: 1) Unidirectional control from sensors to sink; 2) Consider no reliability but congestion control; 3) Result in decreased reliability (although conserving energy) especially under such scenarios with sparse source and high data rate; 4) The delay or response time of closed-loop multi-source regulation will be increased under heavy congestion since the ACK issued from sink would loss with high probability at this time.

Fusion consists of three congestion mitigation techniques [9]. These techniques do not explicitly rely on topology information, it focuses on single-sink, spanning-tree topologies. The first technique is called hop-by-hop flow control, it has two components: congestion detection and congestion mitigation. A simple way to detect congestion is to rely a sensor's queue size. Congestion mitigation is the mechanism by which nodes in a given radio neighborhoods suffocate their transmissions to prevent queues at their next-hop node from overawe. When a sensor overhears a packet from its parent with the congestion bit set. It stops forwarding data, allowing the parent to drain its queues. The second technique is called rate limiting, which meters traffic being admitted to the network to prevent unfairness. There is a natural tendency for the network to deliver traffic originating close to a sink at the expense of traffic sourced deeper inside the network. The third technique is called prioritized MAC, which ensures that congested nodes receive prioritized access to the channel.

\section{OUR CONTRIBUTION}

\subsection{Root cause analysis of the Congestion}

\subsubsection{Input and Output Flow Rate}

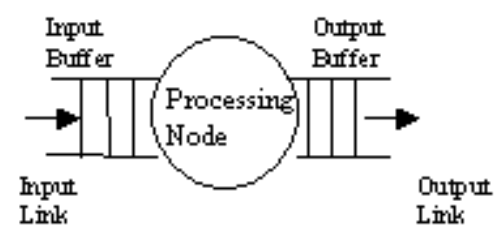

Figure 1. Data packets processing in node

Every node is having its own input and output buffer. This buffer is required for temporary storage of the data packet (see Figure1). As node processes the current packet, next packet is ready for processing. Hence buffer played very important role in storing a temporary packets in queue [5]. This will be maintained by First in First out (FIFO) type of strategy. Because of this temporary storage 'Probability of loss packet' will be reduced.

Input buffer helps to node when incoming packets arrived at current node from previous node, will be stored in input buffer. When particular node is completed its data packet processing, the node pushes processed data packet into the output buffer. If rate of incoming packets is slow, then input buffer will accommodate the enough packets and then it will be given for processing. If processing time is good enough then packets will process and pushed into output buffer in normal flow. When link will free then buffered packets will be hauled into the output link.

The condition becomes worst when input packet flow rate is faster. Node may not be in position to process the incoming data packets at that faster rate. It will take some delay to process it. This will increase the queue in the buffer. If buffer is full then next arrived packets will be dropped or lost. This lost packets probability will be calculated by the Erlang B function. This function is described as follows.

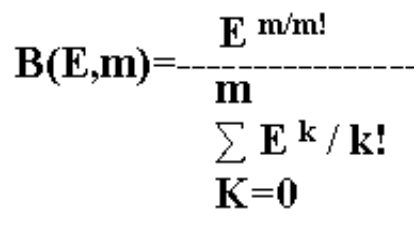

Where B $(E, m)$ is Probability of blocking when

E Erlangs of traffic is offered to $m$ Channels

At the same time when packets are process in node faster than earlier and link will be busy to transmit the data packets, then output buffer will be loaded. This situation will give rise to congestion. It will also increase the backpressure.

\subsubsection{Node density}




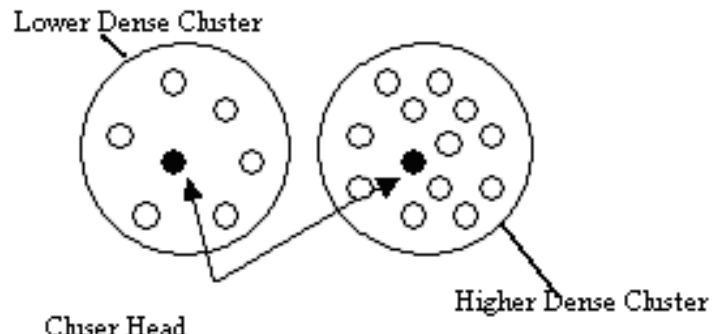

Figure 2. Node Density

When specific requirements are given for the application, based on the specification network designer has to design the clusters. The nodes are placed in the formed clusters depending on the topology selected in the cluster. This process is generally called as 'Localization'. Localization strategy changes according to the application to application. The number of nodes per cluster or unit area is known as 'Node Density'.

Node density [12] plays very important role in congestion control. If cluster has lesser node density, then the probability of finding the neighboring node is less. This is only because the nodes are placed at larger distances from one another. On the other hand if cluster is densely localized then probability of finding the neighboring node is very high.

When probability of finding neighboring node in the denser node cluster, then in case of routing, it is very easier to adopt any routing strategy. In case of lower or lesser dense cluster, the routing becomes more difficult. As far as energy awareness is concern, when the nodes are closer to each other in denser cluster, the node may required less amount of power to transmit to its neighboring node, so energy gets saved. While in less dense cluster, the nodes must equip with higher power radios to transmit data packets for longer distances. As node density increases, traffic towards sink or cluster head get increases which result in the congestion near the sink or cluster heads.

\subsubsection{Non Linear or Unbalanced distribution of Load}

If the nodes are distributed or localized in non-linear fashion in the same cluster, it may result in unbalanced load distribution. This situation may lead to uneven traffic conditions [11], and also the energy is not equally distributed. In the region of lesser density, nodes are required high-energy radios to transmit data packets to far situated neighboring node. On the other side, nodes with enough transmitting capacity radios will serve the purpose. This saves the nodes energy.

This uneven situation in the same cluster creates the differences in the network lifetime of the nodes. The node in the area of lower density will be having less network lifetime and hence died earlier than the region of denser nodes. This is only because denser region nodes required less amount of power to transmit. As far as routing protocols are concern within a cluster, we have to use same protocol for entire cluster. But due to the unbalanced deployment of nodes in a cluster with varying node density, it is very difficult to achieve the same performance in the various regions of the cluster.

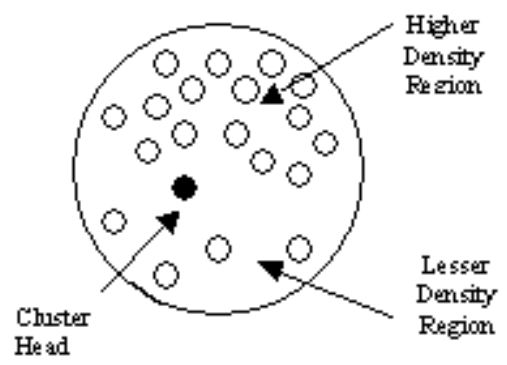

Figure 3. Unbalanced distribution of nodes

\subsubsection{Processing / Service Time of node}

Input Buffer

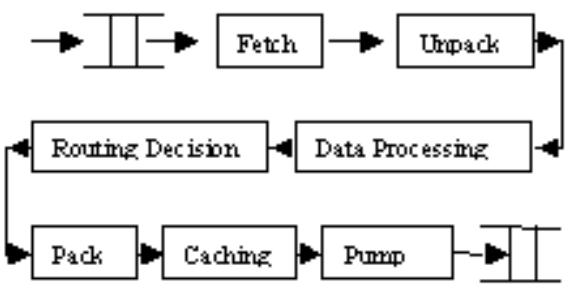

Onflut Buffer

Figure 4. Inner Node processing

Before we discuss the issues of processing the data within node, first we will explore how data get processed within node. Nodes responsibility is to process the data packet, which is queued in the input buffer. Based on the logic of queue packet leave the queue for processing, and get fetched into the node processing zone. Further the packet is unpacked, and data is kept ready for actual processing. Depending on the policy, aggregation will be done. This may include the functions like averaging, summing, etc. The routing strategy will be decided as per networks or cluster policy. The packet is then cached. This is required for hop-by-hop reliable communication strategy. The lost packets may be called by next node to previous node. At this time cached packet will be served to next node. The packet is packed and finally, it is pumped into the output buffer. Above-mentioned process offered for each packet which intern takes some time. Every block in the chain will take time to process the current packet. The main challenge in this case is to reduce the service time to process the packet. The service time depends on the frequency of operation of the processor used in the node. WSN has two types data flow, continues data flow and Event driven data flow. Continues data flows are slow happening. In event driven data flow data packets must be having highest priority. These packets must be reached up to the destination or base station (sink) within less minimum time. Here processing delay plays important role.

If frequency of operation is increased, then the heat is generated by the processor will be larger. On the other hand the power taken from the battery will increase because power consumed by the processor and other allied peripherals will be larger, which reduces the network lifetime of the particular node. If node 
process data with less service time throughput of node will increase and performance of the overall network get increase.

\subsubsection{Reliability of Network}

Sensor Node senses the physical or environmental parameters and forwards the corresponding data to the sink via cluster head. Sometimes nodes are placed in the cluster to serve only routing purpose, these nodes are called as redundant nodes [10]. The nodes that are far away from the sink may require large power radios to transmit the data to sink or other neighboring nodes. The redundant nodes, increases the node density of the cluster. As density of nodes increases in the cluster, the routing performance will increase. The energy utilization become optimum and the network lifetime of the nodes get increase. But the data traffic, which is traveling towards the sink, will also increase, and such situation creates the bottleneck near cluster heads or sink.

The network lifetime of the nodes near sink will decreases, as these nodes are continuously route the accepted data to sink. So the nodes, which are farther distance from the cluster head or sink are having large network lifetime than the nodes near the cluster heads or sink. Finally by using redundant nodes we can increase reliability in network.

\section{CONCLUSIONS AND FUTURE WORK}

Wireless sensor network comprises of deployment of huge amount of sensor nodes with lot of resource constrains, which may cause congestion in the network The parameters described in this paper are some of the root causes of congestion. By monitoring various aspects of WSN such as localization, traffic or load distribution, node processing time etc. we will be able to achieve more reliable network. We are in the process of designing congestion control and avoidance algorithms based on and analyzing the above discussed parameters.

\section{ACKNOWLEDGEMENT}

We would like to welcome the constructive comments from all readers.

\section{REFERENCES}

[1] Chonggang Wang1, Kazem Sohraby1, Bo Li2, and Weiwen Tang Issues of Transport Control Protocols for Wireless Sensor Networks, University of Arkansas, Fayetteville, AR, USA, 72701 The Hong Kong University of Science and Technology, Hong Kong, P.R.China Sichuan Communication Research Planning \& Designing Co., Ltd., Chengdu, China.

[2] Ying Ouyang, Fengyuan Ren, Chuang Lin, Tao He, Chao Li, Yada Hu, Hao Wen A Simple Active Congestion Control in Wireless Sensor Network, Department of Computer Science, Tsinghua University, Beijing, China.

[3] Chieh-Yih Wan, Shane B. Eisenman and Andrew T. Campbell, "CODA: Congestion Detection and Avoidance in Sensor," in Proc. of ACM SenSys '03.
[4] Y. Sankarasubramaniam, O. Akan, and I. Akyildiz, "ESRT: Event-to-Sink Reliable Transport in Wireless Sensor Networks," in Proc. of ACM MobiHoc '03.

[5] Shigang Chen, Congestion Avoidance Based on Lightweight Buffer Management in Sensor Networks, Department of Computer and Information Science and Engineering, University of Florida, Gainesville.

[6] F. Stann and J. Heidemann, "RMST: Reliable data transport in senor networks," in Proceedings of IEEE SNPA'03, May 11, 2003, Anchorage, USA.

[7] C.-Y. Wan, A. T. Campbell, "PSFQ: A reliable transport protocol for wireless sensor networks," in Proceedings of ACM WSNA'02, September 28, 2002, Atlanta, USA.

[9] Bret Hull, Kyle Jamieson, Hari Balakrishnan, Mitigating Congestion in Wireless Sensor Networks, MIT Computer Science and Arti_cial Intelligence Laboratory, The Stata Center, 32 Vassar St., Cambridge, MA 02139

[10] Vehbi Gungor, Ozgur B. Akan, A Reliable Transport (RT) protocol for wireless sensor network \& Actor Networks, IEEE/ACM transaction on Networking; Vol.16.No.2 April 2008

[11]Mark Perillo, Zaho Cheng, and Wendi Heizelman, on the problem of Unbalanced Load Distribution in wireless Sensor Network, Departmental of Electrical and Computer Engineering University of Rochester, Rochester, N71467.

[12] Moslem Noori, Node Density- Characterizing the Traffic Distribution in Linear/ Wireless Sensor Network, Department of Electrical \& Computer Engineering Letters, Vol.12 No. $8^{\text {th }}$ August 2008. 